\title{
Non-Regular Differential
}

\section{Equations and Calculations} of Electromagnetic Fields 
This page is intentionally left blank 


\section{Non-Regular Differential \\ Equations and Calculations \\ of Electromagnetic Fields \\ N E Tovmasyan}

State Engineering University of Armenia Armenia edited by

\section{Z Gevorkyan}

State Engineering University of Armenia Armenia

\section{S Ginovyan}

Institute of Mathematics

Armenian National Academy of Sciences Armenia

\section{N Bobrova}

State Engineering University of Armenia Armenia 


\section{Published by}

World Scientific Publishing Co. Pte. Ltd.

P O Box 128, Farrer Road, Singapore 912805

USA office: Suite 1B, 1060 Main Street, River Edge, NJ 07661

UK office: 57 Shelton Street, Covent Garden, London WC2H 9HE

\section{Library of Congress Cataloging-in-Publication Data}

Tovmasyan, N.E., 1934

Non-regular differential equations and calculations of electromagnetic

fields / N.E. Tovmasyan; edited by L.Z. Gevorkyan, M.S. Ginovyan, M.N. Bobrova.

p. $\mathrm{cm}$.

Includes bibliographical references and index.

ISBN 9810233361 (alk. paper)

1. Electrodynamics -- Mathematics. 2. Differential equations, Partial -- Numerical solutions.

3. Boundary value problems -- Numerical solutions. I. Gevorkyan, L. Z. II. Ginovyan,

M. S. III. Bobrova, M. N.

QC631.T684 1998

537.6'01'515353--dc21

98-10860

CIP

\section{British Library Cataloguing-in-Publication Data}

A catalogue record for this book is available from the British Library.

\section{Copyright $(1998$ by World Scientific Publishing Co. Pte. Ltd.}

All rights reserved. This book, or parts thereof, may not be reproduced in any form or by any means, electronic or mechanical, including photocopying, recording or any information storage and retrieval system now known or to be invented, without written permission from the Publisher.

For photocopying of material in this volume, please pay a copying fee through the Copyright Clearance Center, Inc., 222 Rosewood Drive, Danvers, MA 01923, USA. In this case permission to photocopy is not required from the publisher.

This book is printed on acid-free paper.

Printed in Singapore by Uto-Print 


\section{Preface}

This monograph is devoted to the theory of partial differential equations in the plane domains and their applications in Electrodynamics.

The theory of partial differential equations in the plane domains possesses a number of essential peculiarities. The regular and non-regular, properly and improperly elliptic equations should be distinguished depending on the distribution of the roots of the corresponding characteristic equations. For nonregular and improperly elliptic equations the classical boundary value problems (Cauchy, Dirichlet, Neumann and Poincaré) are not correct.

In this monograph for non-regular differential equations and for improperly elliptic equations we pose and resolve correct boundary problems. The methods developed here are used for investigation of propagation of periodic electromagnetic waves in stratified and non-homogeneous media as well as for calculations of capacitances of a sufficiently wide class of new capacitors.

The book consists of 7 chapters.

In the first three chapters we consider boundary value problems for nonregular partial differential equations in the half-plane. Based on the non-regularity character of these equations we describe and resolve Riemann-Hilbert and Dirichlet-type problems. We describe some efficient methods of resolution of such problems for non-regular differential equations $L_{1} L_{2} \cdots L_{n} u=0$, where $L_{1}, L_{2}, \ldots, L_{n}$ are first-order linear differential operators with constant coefficients.

In chapter 4 we investigate the propagation laws of periodic electromagnetic waves in the stratified and non-homogeneous media. We show that the electric and magnetic intensities can be explicitly expressed by the boundary data.

Chapter 5 is devoted to the calculations of capacitances of cylindrical and spherical capacitors. Using conformal mappings of plane domains we calculate the capacitances of a sufficiently broad class of cylindrical capacitors. Some problems of the best choice of the shape of these capacitors are described and resolved. For spherical capacitors we derive a new simple formula of capacitance. 
In chapter 6 , numerous boundary value problems for improperly elliptic equations in bounded plane domains are investigated. The assumption of impropriety of the equations has essential influence on the description and methods of solution of the problems. For these equations the Riemann-Hilbert and Dirichlet-type problems are of special significance. In the canonical domains (circle, ellipse, etc.), explicit formulae for solutions of these equations are obtained.

In chapter 7 we construct the fundamental systems of solutions for improperly elliptic equations in the class of real-valued functions. The results of this chapter are used in chapter 6 for investigation of boundary value problems for improperly elliptic equations.

Chapters 1, 2, 3 and 7 include the theoretical part of the book, while the chapters 4, 5 and 6 are devoted to the application of the results of theoretical part to the efficient resolutions of concrete applied problems.

The main advantages of this book are:

1. We have constructed classes of functions for which the Riemann-Hilbert and Dirichlet type boundary value problems for non-regular partial differential equations in the half-plane are correct (chapters 1, 2 and 3).

2. We suggest efficient methods of solution of a broad class of boundary value problems for improperly elliptic equations in finite simply connected and canonical domains (chapter 6).

3. The obtained results are applied for investigation of the propagation of periodic electromagnetic waves in stratified and non-homogeneous media, as well as for calculations of capacitances of capacitors with analytic surfaces (chapters 4 and 5).

Methods and results of this book may be applied for resolution of a broad class of important practical problems of mathematical physics that arise in the different fields of science and engineering.

The book is based mostly on the investigations of the author and the larger part of the results is published here for the first time.

The manuscript was read by scientific collaborators of the Department of Mathematics of Armenian State Engineering University and Institute of Mathematics of Armenian National Academy of Sciences. L. Z. Gevorkyan, M. S. Ginovyan, A. O. Babayan and A. A. Andryan have made a great number of useful remarks. Essential assistance in the preparation of the layout of this book was brought by M. N. Bobrova, A. P. Antonyan, A. N. Tovmasyan and S. M. Carapetyan. I would like to thank all of them.

I would like to express a special gratitude to His Holiness Karekin I, Catholicos of All Armenians for the financial support during the preparation of the manuscript. 


\section{Contents}

Preface

1. Dirichlet Type Problems for Non-Regular Differential Equations in the Half-Plane

1.1. Description of the Problem and Main Results $\ldots \ldots \ldots \ldots \ldots \ldots \ldots 1$

1.2. Some Auxiliary Propositions .......................... 4

1.3. Cauchy Problem for Equation (1.1) in the Class of Analytic Functions .................................... 11

1.4. Dirichlet Type Problem for Equation $(1.1) \ldots \ldots \ldots \ldots \ldots \ldots \ldots, 16$

1.5. Examples ...................................... 21

2. Riemann-Hilbert Problem for a Class of Non-Regular Elliptic Equations

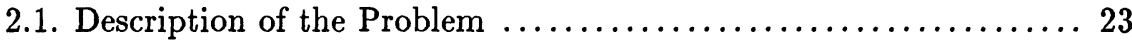

2.2. The General Solution of Equation $(2.1) \ldots \ldots \ldots \ldots \ldots \ldots \ldots \ldots \ldots$

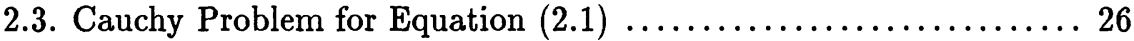

2.4. Riemann-Hilbert Problem for Analytic Functions ............ 28

2.5. Investigation of the Problem $(2.1),(2.2) \ldots \ldots \ldots \ldots \ldots \ldots \ldots \ldots \ldots \ldots \ldots$

2.6. General Boundary Value Problems for Equation $(2.1) \ldots \ldots \ldots 32$

2.7. Exceptional Cases for Problem (2.1), (2.2) ............... 33

2.8. Correct Boundary Value Problems for Equation (2.1) in General ......................................... 41

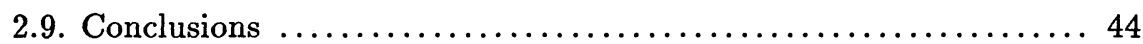

3. Dirichlet Type Problem for the Product of First Order Differential Operators

3.1. Description of the Problem and Main Results .............. 45

3.2. The General Solution of Non-Homogeneous Elliptic Equation ... 46

3.3. Cauchy Problem for Equation $L_{1} \cdots L_{r} u=0 \ldots \ldots \ldots \ldots \ldots \ldots$

3.4. Riemann-Hilbert Problem for Equation (3.2) $\ldots \ldots \ldots \ldots \ldots \ldots \ldots$ 
3.5. The Existence of Solution of the Problem (3.2), (3.6), (3.7) _... 52

3.6. The Uniqueness of Solution of the Problem (3.2), (3.6), (3.7) ... 54

3.7. Dirichlet Problem for Second Order Elliptic Equations ........ 57

3.8. Dirichlet Problem for 2m-th Order Elliptic Equations .........6 61

3.9. Riemann-Hilbert Problem for Paired Elliptic Equation ......... 63

3.10. Dirichlet Problem for Generalized Analytic Functions ......... 66

3.11. Correct Boundary Value Problems for Products of First

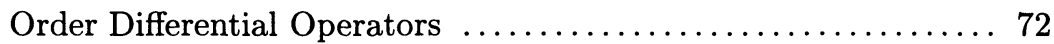

3.12. Normal Solvability of Dirichlet-Type Problem for Products of First Order Differential Operators $\ldots \ldots \ldots \ldots \ldots \ldots \ldots \ldots \ldots . \ldots$

3.13. A Method to Solve Cauchy Problem for Elliptic and Hyperbolic Equations $\ldots \ldots \ldots \ldots \ldots \ldots \ldots \ldots \ldots \ldots \ldots \ldots \ldots \ldots$

4. Propagation of Plane Periodic Electromagnetic Waves in Stratified Medium

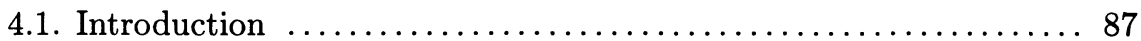

4.2. Boundary Value Problem for the System (4.4) - (4.6) in Homogeneous Medium

4.3. A General Boundary Value Problem for the System (4.10) in Homogeneous Conducting Medium

4.4. Boundary Value Problems for the System (4.10) in $x>0$ Consisting of Two Homogeneous Strata

4.5. On Fredholmity of General Boundary Value Problem for Equation (4.10) in Two Strata Medium $x>0 \ldots \ldots \ldots \ldots \ldots . \ldots 110$

4.6. Harmonic Oscillations of Electromagnetic Waves in Multi-

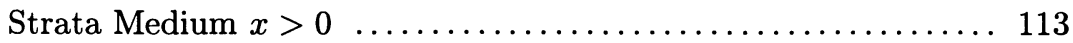

4.7. Harmonic Oscillations of Electromagnetic Waves in NonHomogeneous Media

\section{Calculation of Capacitances of Cylindrical and Spherical Capacitors}

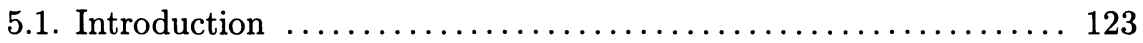

5.2. Invariance of Capacitances under Conformal Mappings ........ 124

5.3. Formulae of Capacitances with Cross-Sections Bounded by Analytic Curves ................................ 127

5.4. Some Tests of Equality for Capacitances ................. 136

5.5. A Method of Definition of Capacitance for Spherical Capacitors .................................. 141

5.6. Approximative Formulae for Capacitance ............... 149

5.7. Optimal Choice of Cylindrical Capacitor's Shape ........... 154 
6. Efficient Methods of Solution of Boundary Value Problems for Improperly Elliptic Equations

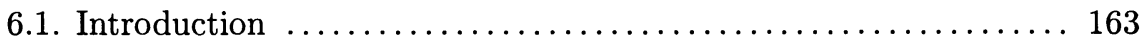

6.2. Some Auxiliary Propositions ......................... 165

6.3. Riemann-Hilbert Type Problem for a Class of Improperly Elliptic Equations ................................ 168

6.4. Dirichlet Type Problem for Improperly Elliptic Equations ...... 173

6.5. Riemann-Hilbert Problem for Second-Order Improperly Elliptic Equations in Simple Connected Domains ........... 176

6.6. Dirichlet Type Problem for Third-Order Improperly Elliptic Equations ................................ 183

6.7. Riemann-Hilbert Problem for Second-Order Improperly Elliptic Equations in the Circle ..................... 188

6.8. Riemann-Hilbert Problem for High Order Improperly Elliptic Equations ............................... 194

6.9. Dirichlet Type Problem for High Order Improperly Elliptic Equations ............................... 195

6.10. Neumann Type Problem for Improperly Elliptic Equations . . . . 199

6.11. Poincaré Problem for Bitzadze Equation ................ 205

7. Some Classes of Improperly Elliptic Equations 213

7.1. Improperly Elliptic Equations in a Class of Real-Valued

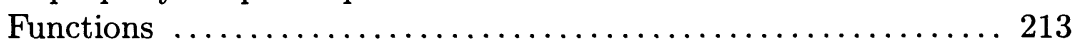

7.2. Two Elliptic Equations with One Unknown Function ........ 220 Bibliography $\ldots \ldots \ldots \ldots \ldots \ldots \ldots \ldots \ldots \ldots \ldots \ldots \ldots \ldots \ldots \ldots .229$

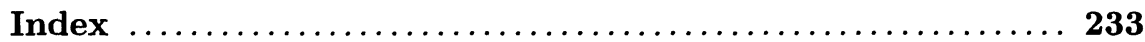

